\title{
EDEM Simulation Study of Lignite Pressing Molding with Different Particle Size
}

\author{
Na Zhao ${ }^{\mathrm{a}}$, Fang Zhao ${ }^{\mathrm{b}}$ and Wen-zhen Zhong ${ }^{\mathrm{c}}$ \\ School of Mechanical Engineering, Jinan University, Jinan 250022, China. \\ azhaonajndx@163.com, ${ }^{b}$ me_zhaof@ujn.edu.cn, ${ }^{\mathrm{C}} 546508266 @ q q . c o m$
}

Keywords: Lignite, Pressure molding, Particle diameter, EDEM.

\begin{abstract}
Based on the discrete element software EDEM, the bond model is created and introduced to realize the dynamic simulation of the whole process of the material entering the roller surface and being pressed. The analysis of pressing four particle size lignite at two speed shows that the diameter of a ball made of single particle size lignite particle is reduced with the increase of particle size; the smaller the particle size of lignite pressed molding is, the more particles are in the ball, which are more compact.
\end{abstract}

\section{Introduction}

Lignite is a young coal of the lowest degree of coalification, easy weathering and spontaneous combustion, low heat quantity, high moisture content, only through screening, washing, molding and other primary processing, lignite is transported and stored to meet the requirements of fuel and raw users. At present, rolling ball machine [1] is the main equipment of ignite pressing and shaping, its structure simple, lower cost, widely used in all kinds of powder molding field [2].

Lignite pressure molding is to compress lignite into the coal products of a certain shape and strength under the condition of a binder or without by a mechanical method [3]. For rolling ball machine, pressing molding is lignite can become a ball that achieves the desired size, with certain compactness and the bonding force enough. So, as a bulk material with special character, lignite particle size and its distribution become one of the important factors affecting pressing forming, which need to study on the interaction rules between coal particles from the perspective of mesoscopic and discrete.

The discrete element method (DEM) is a powerful tool for analyzing the deformation and motion trend of discrete media. In 1971, after Cundall proposed a discrete element method for rock mechanics, the study of particle discrete element is developing rapidly, and used in various fields of geotechnical, mining, chemical and so on [4], some convenient and practical software tools are generated. EDEM software is a CAE software based on discrete element method to simulate and analyze the process treatment and production operation of particle system. In this paper, the software is used to simulate the molding process of powder coal, and to analyze the behavior of different particle size and its influence on the molding effect in the process of pressing.

\section{The Modeling Thought of Particle Bonding Model}

The modeling thought of particle bonding model is that lignite molding is regarded as the process of particle bonding, the cohesive force and compaction degree increasing through external force. When using discrete element software EDEM to do simulation analysis, in order to simulate the bond and shaping of the material, the API contact bonding procedure is required. Bonding is calculated by using energy density value multiplied by overlapping area, adding *nTime product coefficient when creating the bond model plug-in, this will make the viscosity increase with time increasing, and cohesive force between particles also increases. Cohesiveness includes the bonding among the particles which are related to time, and among the particles and geometry. EDEM software provides application programming interface (API), so, a functional module carried with by non EDEM system can be developed. 
In order to simulate the process of lignite compacting and molding, particle factory of software EDEM is used to produce of a number of small spherical particles, particle size is set by coal particle size in the actual production to make it more consistent with the real situation, at this moment, there is no adhesion between small particle balls. Under the condition of gravity, these small spherical particles fall into the half round ball of the roller surface. After forming stable granular material accumulation, they will enter the extruding zone of double roller; lastly, the two rollers rotate oppositely to extrude small particles. At the same time, the created particle plug makes the bonding force between particles increase with time. And through the common effects of the roller pressure and the cohesive force between particles, bonding between particles will be formed, and small particles bonded together with the help of bonding. A certain amount of overlap will be formed between every two adjacent small particles, and the amount of overlap increasing implies that the adhesive force also increases and the adhesive force is changed according to the actual physical property. Particles bonding as shown in figure 1, figure 1 (left) shows particles going into the simulation area to bond. Here are the only some particles captured to indicate, picture on the right is a mass of bonded particles following the Picture 1 . The whole picture represents bonding effect visually in the process of EDEM simulation.
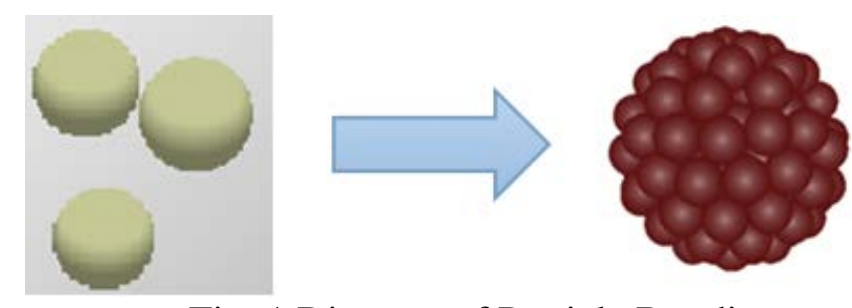

Fig. 1 Diagram of Particle Bonding

\section{The Constraint Setting}

EDEM can assume the properties of the particles, and describe the particles shape accurately by introducing the real model, and create a particle model by adding mechanical properties, material properties and other physical properties, and save data into the appropriate database during the simulation.

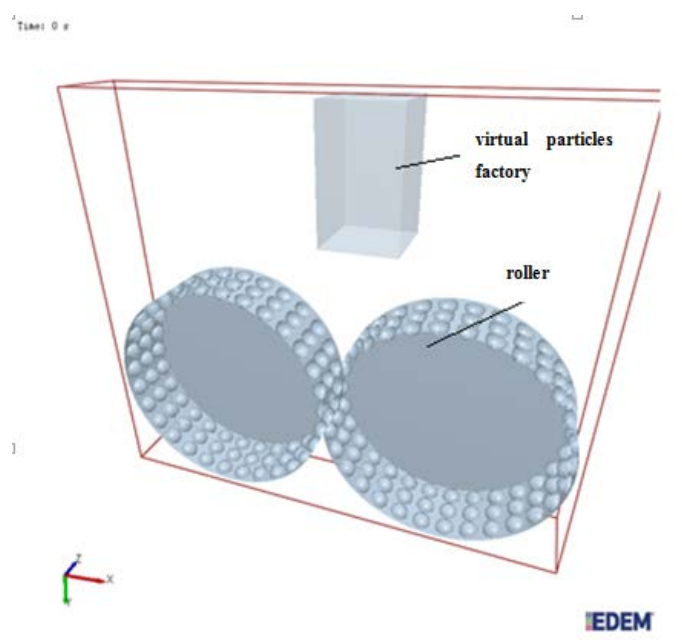

Fig. 2 the Roller Model in EDEM

Using SolidWorks 3D software to model the rollers of pressure ball machine, and the roller diameter is $200 \mathrm{~mm}$, and in order to reduce the simulation time, the ball socket is set to stand in three rows, the ball socket is a hemisphere of $20 \mathrm{~mm}$ diameter. As shown in Figure 2, importing the model into EDEM through IGS format to set the constraints. Importing the model into discrete element simulation software, and setting the physical attributes of particle to particle and particle to geometry,the gravitational acceleration along the $y$-axis is $9.81 \mathrm{~m} / \mathrm{s}^{2}$. According to the data, material properties of the particles: Poisson ratio is 0.3 , shear modulus is $2.3 \mathrm{e}+9$, density is $1200 \mathrm{~kg} / \mathrm{m} 3$. In 
globals boundary, setting interaction coefficient of particles and particles, particles and rollers, of which particulate material recovery coefficient is 0.25 , coefficient of static friction is 0.2 , coefficient of rolling friction is 0.14 , and the recovery coefficient between particles and the rollers material is 0.5 , coefficient of static friction is 0.5 , coefficient of rolling friction is 0.01 . To simulate the actual working conditions, create a virtual feeder, roller speed will be $10 \mathrm{rpm}$. In addition, the time window of $1 \mathrm{~s}$ is set up to observe several continuous process of lignite pressing into the ball, and to obtain a reasonable and stable simulation data.

\section{The Data Analysis of Simulation Results}

The Simulation results visualization

Figure 3 shows the particles state at the beginning of simulation in the period of 0 s- 0.1 s, as can be seen from the chart, in this period of time ,the particles which fall into the roll ball socket from the Particles factory, are in a stable state did not change significantly.

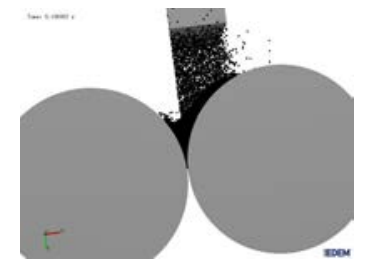

Fig. 3 Particles Fall into Ball Socket

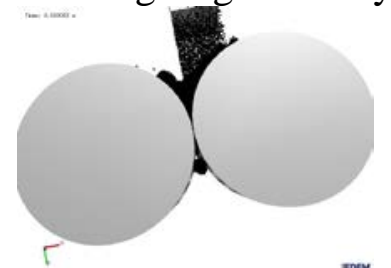

Fig. 4 Particles are Squeezed into a Ball

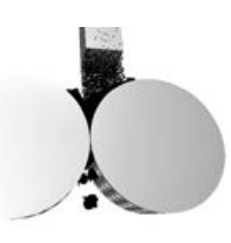

Fig. 5 Pall Out

Figure 4 shows the particles state during the simulation in the period of $0.2 \mathrm{~s}-0.5 \mathrm{~s}$, in the picture, the particles can be seen to enter the extrusion process area, and then to be pressed, and then pressed into a ball.

Figure 5 shows the particles state during the simulation in the period of $0.5 \mathrm{~s}-1 \mathrm{~s}$, it can be seen from the figure, the degree of particles bonding is enough to prevent the particles dispersion,when the particles are out of the ball. And the all particles of a ball can be shaped into an entity.

Figure 6 shows the contact curve of the particles and the particles.The abscissa indicates the simulation time, the vertical axis represents the total number of contact particles. as shown in figure 6 , as the time goes on, the number of contact particles starts to increase rapidly, then increase slowly at an inflection point (ball and socket begun to squeeze), this indicates that the bonding force of the particles in the ball increases gradually.

Figure 7 shows the contact curve of the particles and the roller surface (including the ball sockets surface and the plane between ball sockets). As shown in figure, the particles from falling into the roller ball socket to be in beginning of to be squeezed, the number of particles in contact with the roller surface gradually increases before 0.4 seconds. Between 0.5 and 0.6 seconds, particles experience a process that they are squeezed into a ball and then are off the ball, therefore, the number of particles in contact with the roller surface increase firstly, as the particles are squeezed, then decline with forming a ball and out of the ball sockets. Between 0.6 and 0.8 seconds, the motion of particles and the number of particles in contact with the roller surface are similar to that of 0.5 seconds to 0.6 seconds. Thus the number of particles in contact with the roller surface presents wavy curves of increasing, decreasing and increasing respectively as shown in figure 7.
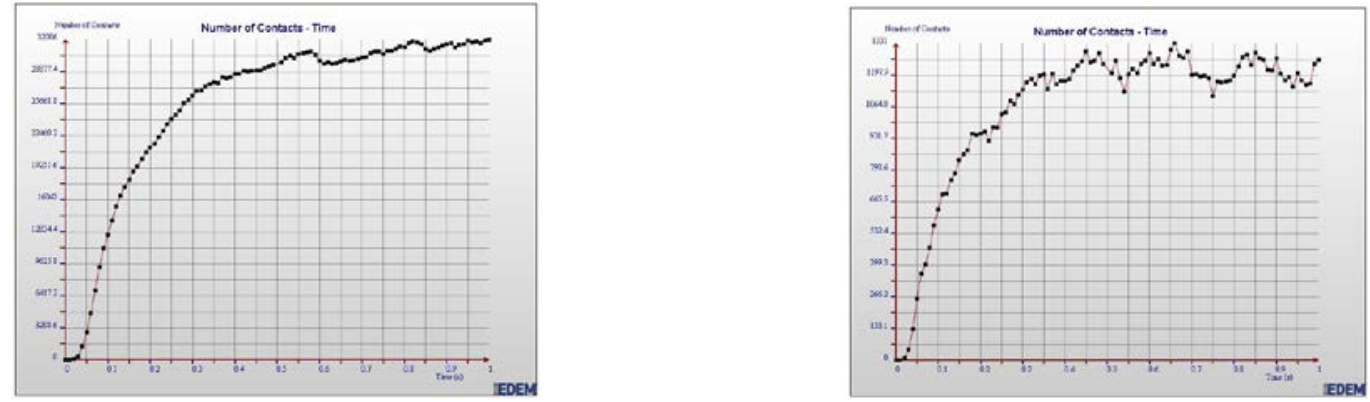

Fig. 6 Contact Curve of the Particles and Particles Fig. 7 Contact Curve of Particles and Roll Surface 


\section{The Effect of Particle Size on the Formation of Lignite}

Using EDEM software, this paper simulates the process of the particle pressing of four kinds of particle size, $2 \mathrm{~mm}, 2.5 \mathrm{~mm}, 1.5 \mathrm{~mm}$ and $3 \mathrm{~mm}$ respectively, and the forming effect is analyzed from three aspects: whether a ball being shaped or not, the ball size, the number of particles in the ball. Figure 8 to Figure 11 are the four particle size of lignite formation when roller rotation speed is 10 rpm. As can be seen from the comparison of four pictures, they all can be pressed into a ball, and in the process of the lignite pressing, with the increase of the particle size, the number of particles bonded into a ball is reduced. Four kinds of particle size were pressed into a ball and the ball diameter is shown in Table 1 , respectively $18.08 \mathrm{~mm}, 16.84 \mathrm{~mm}, 15.07 \mathrm{~mm}, 14.23 \mathrm{~mm}$, that with the increase of particle size, the ball diameter is reduced.

Figure 12 to 15 are line chart that the number of particles of a ball varies with time when four kinds of lignite particle size in the process of forming, as you can see, the number of particles of bonding each other in ball socket showing a rising trend with the time going on,in other words, as time increases, the number of particles bonded are becoming more and more. But when the four size of particles are bonded into a ball by each oneself, the number of particles in the ball is different, and the number of particles bonded to the ball is reduced with the increase of the particle size, and the number of particles bonded into a ball during lignite particles of $1 \mathrm{~mm}$ forming is more than when lignite particles of $2 \mathrm{~mm}$ forming, and when lignite particles of $1 \mathrm{~mm}$ forming, the number of particles bonded is the smallest. Using EDEM software can extract the total particle number that different particle size of coal particles in forming and bonding into a ball in a simulation time (eg. 1s), and the number of particles can be obtained through the calculation included a ball, as shown in Table 1. For comparison, it is assumed that every ball filled with particles and every two adjacent particles just contact (no overlap, also no clearance), seeing this situation as critical situation. Lignite Particle size of $1.5 \mathrm{~mm}, 2$ $\mathrm{mm}, 2.5 \mathrm{~mm}$ after being pressed into the ball, each contain particles whose number is larger than the number of particles of critical situation, and the percentage value between the former and the latter as shown in Table 1. It can be seen that the delta value is reduced with the increase of particle size, indicating when the smaller size particle of lignite are compressed into a shape, the ball contains more particles, that is the more dense molding.

From analysis can be known, during the small particles of coal particles molding, under the appropriate pressure, the particle contact area is large and they are easy to reunite and meshing [5]. When the large granular coal granule molding, coal grain total surface area is small, the degree irregularity of large particles of coal particles and unevenness is large, there is loosing contact between the coal particle, it is not easy to form adhesion and cohesion, and there is large gap when larger particles of pulverized coal are pressed into the ball, so, the number of particles bonded to a ball is less. Therefore, the particle size of a single particle lignite in the pressing and molding, the smaller lignite particle in pressing and forming into a ball is more easily, and the diameter of the ball is large, the number of particles in the ball is larger. Thus, the forming is more dense and the effect will be better.

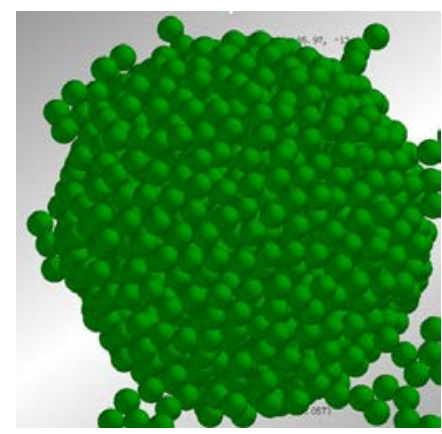

Fig. 9 1.5mm Bonded Particles Forming

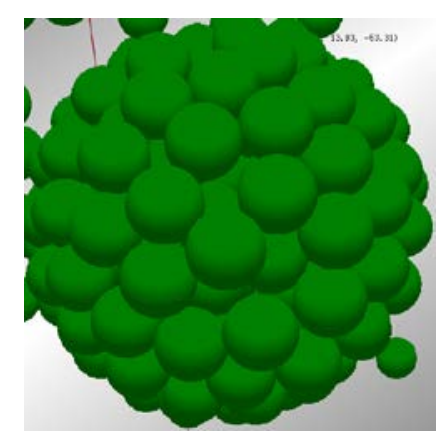

Fig. 10 2mm Bonded Particles Forming 


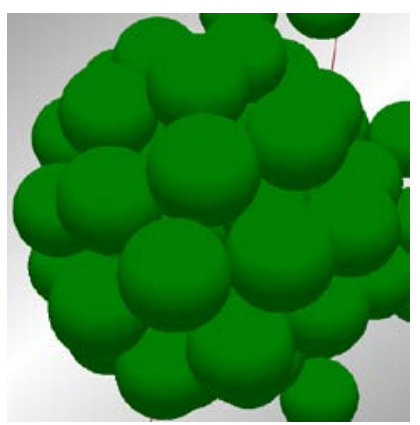

Fig. 11 2.5mm Bonded Particles Forming

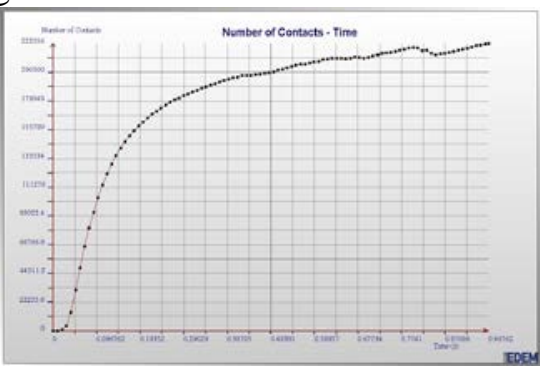

Fig. 13 The number of 1.5mm Particle Bonding

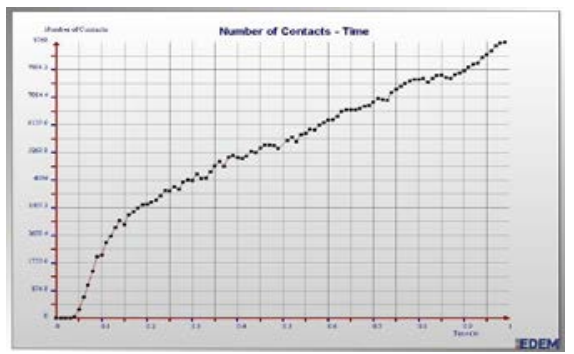

Fig. 15 The number of 2.5mm Particle Bonding

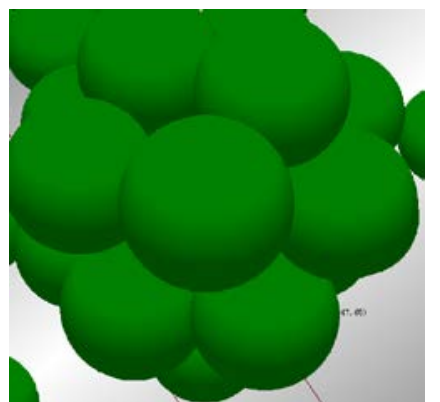

Fig. 12 3mm Bonded Particles Forming

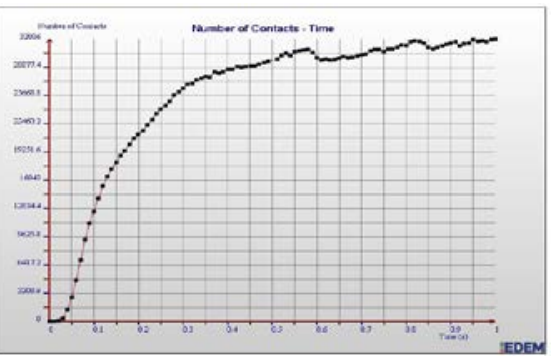

Fig. 14 The number of 2mm Particle Bonding

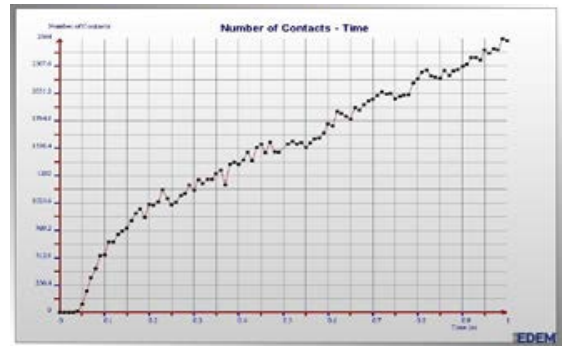

Fig. 16 The number of 3mm Particle Bonding

Table 1 Simulation Results of Different Particle Size Repression under Roller Speed of 10rpm

\begin{tabular}{ccccc}
\hline $\begin{array}{c}\text { Particle } \\
\text { diameter } \\
(\mathrm{mm})\end{array}$ & $\begin{array}{c}\text { Ball diameter } \\
(\mathrm{mm})\end{array}$ & $\begin{array}{c}\text { The number of } \\
\text { particles in a ball }\end{array}$ & $\begin{array}{c}\text { The number of } \\
\text { particles in the } \\
\text { critical situation }\end{array}$ & Percentage value \\
\hline 1.5 & 18.08 & 14000 & 3000 & $366 \%$ \\
2 & 16.84 & 2139 & 1000 & $114 \%$ \\
2.5 & 15.07 & 584 & 512 & $14 \%$ \\
3 & 14.23 & 200 & 296 & $-32 \%$ \\
\hline
\end{tabular}

Simulation results of different particle sizes repression under roller speed of 20rpm as shown in Table 2.

Table 2 Simulation Results of different Particle Size Repression under Roller Speed of 20rpm

\begin{tabular}{ccccc}
\hline $\begin{array}{c}\text { Particle } \\
\text { diameter } \\
(\mathrm{mm})\end{array}$ & $\begin{array}{c}\text { Ball diameter } \\
(\mathrm{mm})\end{array}$ & $\begin{array}{c}\text { The number of } \\
\text { particles in a ball }\end{array}$ & $\begin{array}{c}\text { The number of } \\
\text { particles in the } \\
\text { critical situation }\end{array}$ & Percentage value \\
\hline 1.5 & 16.73 & 9417 & 3000 & $214 \%$ \\
2 & 15.17 & 1850 & 1000 & $85 \%$ \\
2.5 & 14.03 & 439 & 512 & $-14 \%$ \\
3 & 13.82 & 139 & 296 & $-53 \%$ \\
\hline
\end{tabular}

As can be seen from table 2, when the roller is at the speed of 20rpm, the ball diameter decreases with particle size becoming bigger, and the number of the particles in the ball decreases with particle size becoming bigger. That more smaller the lignite particle size is in press molding, the more easily a ball is formed, and the diameter of the ball is bigger, the ball contains more particle, so the shape is 
more compact, and the effect will be better. Thus it has the same conclusion with roller speed of 10 rpm. By comparing the corresponding data in table 1 and table 2, when the roller speed is $20 \mathrm{rpm}$, the ball diameter and the number of particles contained in a ball are less than roller speed for $10 \mathrm{rpm}$. That is, the effect of the ball at the speed of 10rpm is better than the speed of 20rpm, and this is related to the roller speed increasing, the material between two rollers stays less time, and being short of the effect of the pressure, they will can not be properly achieved sufficiently deforming and overlapping of particles.

\section{Conclusion}

Using EDEM software and adding bonding model, the process of lignite particles pressing into a ball can be simulated.

When pressing lignite of a single particle diameter, with particle size increasing, the diameter of a ball pressed is reduced.

The smaller the particle size of lignite pressed into a ball is, the more particles are in the ball, which are more compact.

\section{References}

[1] Hu Ruixia. Metallurgical sludge and waste Utilization Research [J] Chemical Engineering \& Equipment, 2008 (1): 60.

[2] Zhang Ping, based on microscopic observation and image processing coal particles detection [D]. Huazhong University of Science and Technology, 2004.

[3] Deng Jiayao, Zhang Huiqiang, Wu Jian, et al. Effect of particle size, pressure and moisture of coal cold intensity [J]. Journal of Engineering Thermophysics. 2004 (S1).

[4] Yang Yamin, Zhao Fang, Duan Derong, et al.The Accelerate Effect of Material Particle Size of the New VSI Rotor Based on EDEM[J].Equipment manufacturing technology, 2012(8).

[5] Lin Yu, Liu Shuling, Lin Qun. Study on the strength of compaction coal logs[J].Journal of china coal society 2005,30(2):241-244. 